Published in final edited form as:

ACS Appl Mater Interfaces. 2019 July 10; 11(27): 23893-23900. doi:10.1021/acsami.9b06453.

\title{
In Vivo Assessment of Decellularized Porcine Myocardial Slice as an Acellular Cardiac Patch
}

\author{
Mickey Shah ${ }^{\dagger,}$, Pawan KC $\ddagger$, Ge Zhang ${ }^{*}, \ddagger$ \\ †Integrated Bioscience Program, The University of Akron, Akron, Ohio 44325-0302, United States \\ ‡Department of Biomedical Engineering, The University of Akron, Akron, Ohio 44325-0302, \\ United States
}

\begin{abstract}
Acellular cardiac patches made of various biomaterials have shown to improve heart function after myocardial infarction (MI). Extracellular matrix scaffold derived from a decellularized tissue has unique advantages to serve as an acellular cardiac patch due to its biomimetic nature. In this study, we examined the therapeutic outcomes of using a decellularized porcine myocardium slice (dPMS) as an acellular patch in a rat acute MI model. dPMSs with two different thicknesses (300 and $600 \mu \mathrm{m}$ ) were patched to the infarcted area of the rat myocardium, and their effects on cardiac function and host interactions were assessed. We found that the implanted dPMS firmly attached to host myocardium after implantation and prevented thinning of the left ventricular (LV) wall after an MI. A large number of host cells were identified to infiltrate into the implanted dPMS, and a significant number of vessel structures was observed in the dPMS and infarcted area. We detected a significantly higher density of M2 macrophages in the groups treated with dPMSs as compared to the MI group. Contraction of the LV wall and cardiac functional parameters (left ventricular ejection fraction and fractional shortening) was significantly improved in the treatment groups (300 and $600 \mu \mathrm{m}$ dPMS) 4 weeks after surgery. Our results proved the therapeutic benefits of using dPMS as an acellular cardiac patch for the treatment of acute myocardial infarction.
\end{abstract}

\section{Graphical Abstract}

\footnotetext{
*Corresponding Author: gezhang@uakron.edu. Phone: 330-972-5237. Fax: 330-374-8834.

Supporting Information

The Supporting Information is available free of charge on the ACS Publications website at DOI:10.1021/acsami.9b06453. Characterization of decellularized porcine myocardial tissue; presence of inflammatory cells; echocardiographic data (PDF) 


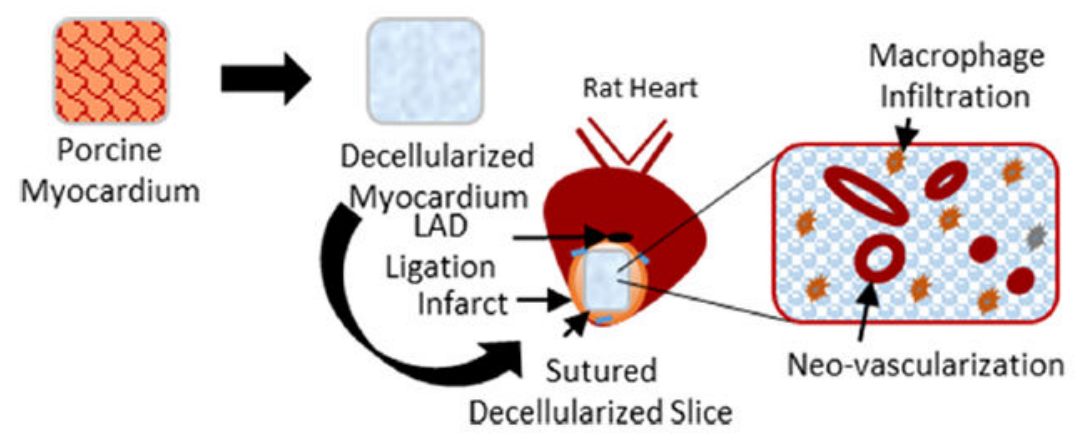

\section{Keywords}

acellular cardiac patch; decellularized extracellular matrix; rat MI model; vascularization; heart remodeling

\section{INTRODUCTION}

Acute myocardial infarction (MI) can lead to irreversible damage to myocardial tissue and heart function. The massive loss of cardiomyocytes during an MI initiates the formation of scar tissue due to the insufficient regenerative capability of adult myocardium. This results in ventricular wall thinning and eventually leads to heart failure. ${ }^{1,2}$ Current clinical treatments of MI are incapable of completely preventing adverse ventricular remodeling after MI. ${ }^{3}$ Cardiac patches aim to supply cells and/or therapeutic agents to repair the injured myocardium and provide mechanical support to help with the recovery of heart function..$^{4,5}$ Compared with cellular patches, acellular cardiac patches have unique advantages such as (1) avoiding the complications of exogenous cell transplantation (e.g., poor cell survival and engraftment rate, triggered immune response, high cost), ${ }^{6-8}$ (2) they can be implanted immediately because of their off-the-shelf availability, and (3) they allow for the implementation of various tissue engineering strategies for myocardial repair (e.g., recruiting host cells, growth factor delivery, mechanical compensation).

Extracellular matrix (ECM) scaffold has shown great promise in serving as an acellular cardiac patch. ECM scaffold can be obtained via tissue decellularization to remove the cellular component and retain the native structure and composition of ECM. ${ }^{9}$ Following a 3[(3-cholamidopropyl)dimethylammonio]-1-propanesulfonate (CHAPS)-based decellularization protocol, decellularized rat lung scaffold has demonstrated a similar extracellular matrix (ECM) composition and mechanical properties as a native lung. ${ }^{10} \mathrm{~A}$ preserved vascular tree has been observed in decellularized cardiac ECM. ${ }^{11}$ Various ECM components, cytokines, and cell binding ligands have been proven to remain in the ECM scaffold after decellularization, which provide a tissue-like environment for cell attachment and interaction. ${ }^{12}$ Furthermore, promising outcomes have been reported when using acellular ECM scaffolds to treat MI. ${ }^{13}$ The ECM scaffolds derived from the porcine small intestinal submucosa (SIS), when employed as an acellular cardiac patch, have induced angiomuscular regeneration in a porcine MI model. ${ }^{14}$ Acellular cardiac patches made of 
decellularized epicardium have also contributed to post-MI heart function recovery in a rat MI model. ${ }^{15}$

Our previous in vitro studies have demonstrated the advantages of using decellularized porcine myocardium slice (dPMS) as a cardiac patch. ${ }^{16}$ We have shown that dPMS supports cell attachment, viability, and infiltration while still maintaining the cardiac tissue mechanical characteristics. In this study, we further assessed the therapeutic outcomes of dPMS as an acellular patch using a rat acute MI model. dPMSs with two different thicknesses (300 and $600 \mu \mathrm{m}$ ) were patched to the infarcted area of the rat myocardium, and their effects on cardiac function and host interactions were examined.

\section{MATERIALS AND METHODS}

\subsection{Preparation of Decellularized Porcine Myocardium Slices (dPMS).}

Thin dPMS slices were prepared, as described previously. ${ }^{16}$ Briefly, fresh myocardial pieces were isolated from the anterior left ventricular wall of pig hearts. Myocardial pieces were washed with water to remove blood, and decellularization was carried out by $1 \%(\mathrm{w} / \mathrm{v})$ sodium dodecyl sulfate with $0.5 \%$ antibiotics (penicillin/streptomycin) for 2.5 weeks with gentle agitation at room temperature. The fresh solution was changed every day. After the decellularization process, the tissue was rinsed with $1 \times$ phosphate-buffered saline (PBS) for 3 days and stored finally at $-20{ }^{\circ} \mathrm{C}$ until use. Decellularized porcine myocardium slices (dPMSs) with a thickness of either 300 or $600 \mu \mathrm{m}$ were obtained by cryosectioning the decellularized tissue and used as acellular patches for in vivo studies.

\subsection{Rat MI Model and Cardiac Patch Transplantation.}

The Animal Care and Use Committee (IACUC) protocol approval and guidelines were followed for all of the animal housing, surgery, and procedures at the University of Akron (14-11-14-ZRD and 17-10-12 ZRD). Male Sprague Dawley rats (8 weeks old) weighing 250-300 g were purchased (Envigo) and used to create the acute MI model as follows. Rats were anesthetized with ketamine $(80 \mathrm{mg} / \mathrm{kg})$ and xylazine $(5-10 \mathrm{mg} / \mathrm{kg})$ by peritoneal injection, followed by endotracheal intubation. Mechanical ventilation was achieved by connecting the endotracheal tube to the ventilator (HARVARD 663) cycling at 80 breaths per minute and a tidal volume of $1.2 \mathrm{~mL}$ per $100 \mathrm{~g}$ body weight. The heart was accessed via left thoracotomy between the third and fourth intercostal spaces. The pericardium was opened, and the left anterior descending (LAD) coronary artery was permanently ligated using a 6-0 polypropylene suture (Ethicon) to induce an MI. For the sham group, the suture was passed around LAD artery without ligation. For the treatment group, after ligation, 300 or $600 \mu \mathrm{m}$ dPMS was patched to the surface of the infarcted area of the left ventricle by suturing three points around the border. After implantation, the chest was closed, and the animal was left to recover. All animal surgeries were performed under loupe magnification by skilled technicians with extensive surgical experience to ensure the precision of the procedure and avoid animal death after patch implantation. After surgery, 68 rats were randomly assigned into MI only ( $n=18), 300 \mu \mathrm{m}$ dPMS on MI ( $n=18), 600 \mu \mathrm{m}$ dPMS on MI $(n=18)$, or the sham group $(n=9)$. Animals from all groups were euthanized, and the hearts were harvested for evaluation after week 1 , week 2 , or week 4 of surgery. 


\subsection{Cardiac Function Assessment.}

Transthoracic echocardiography was used to assess the cardiac function. M-mode images were obtained from the parasternal short axis view, mid-papillary muscle level using a Vevo 2100 High-Resolution echocardiography Imaging System (VisualSonics, Toronto, Ontario, Canada) designed for small animal studies. After the induction of anesthesia (3\% isoflurane with $100 \%$ oxygen, $1 \mathrm{~L} / \mathrm{min}$, in a small chamber), rats were placed on a controlled heating table designed for small animal echocardiography. The anesthesia and oxygen were delivered through the nose cone (1-2\% isoflurane, $0.5 \mathrm{~L} / \mathrm{min}$ with oxygen). After removing hair from the chest, warmed $\left(37^{\circ} \mathrm{C}\right)$ Aquasonic 100 gel (Parker Laboratories, Fairfield, NJ) was placed on the chest to optimize visibility of cardiac structures. Cardiac function was measured at 2 days before and up to 4 weeks after surgery. Left ventricular end diastolic volume (LVEDV) and end systolic volume (LVESV) as well as left ventricular internal diameter at end diastole (LVID,d) and end systole (LVID,s) were measured. Left ventricular volume was calculated using the modified Teichholz formula: $\operatorname{LVV}=(7.0 /(2.4+$ LVID $)) \times$ LVID. Left ventricular ejection fraction (LVEF\%) was calculated as: ((LVEDV - LVESV)/ LVEDV $) \times 100$. Fractional shortening $(F S \%)$ was calculated as: ((LVID,d - LVID,s)/ LVID,d $) \times 100$. All echocardiographic calculations and measurements were performed offline using the Vevo Lab 3.1.1 software by a double-blinded ultrasonographer and technician. All measurements were averaged over five cycles, and five animals were used for each group.

\subsection{Histology Analysis.}

Animal hearts were isolated after euthanasia at the planned time points. Harvested hearts were washed with $1 \times$ PBS and transversely cut into upper, middle, and lower parts $(\sim 5 \mathrm{~mm}$ each) using a heart slicer. Tissues were washed again with $1 \times$ PBS and fixed into $4 \%$ paraformaldehyde at room temperature. Fixed tissues were processed, dehydrated, and embedded in paraffin blocks. Multiple sections were obtained along the infarcted heart (with/without patch) at $7 \mu \mathrm{m}$ thickness and stained with hematoxylin and eosin (American MasterTech Scientific) according to manufacturer's recommendations. Sections were also stained with Masson's Trichorme (Sigma-Aldrich) to observe the infarcted region of the heart. The LV wall thickness was measured at the minimum thickness region of the infarcted LV wall including applied dPMS. Three sections in each heart were counted and averaged for six hearts in each group.

For immunofluorescence staining, the previously established protocol was followed. ${ }^{16}$ Briefly, paraffin-embedded tissue sections were deparaffinized and rehydrated. The heatmediated antigen retrieval was performed using $1 \times$ citrate buffer ( $\mathrm{pH}$ 6.0), followed by permeabilization and goat serum blocking. Sections were incubated with primary antibody for $18-22 \mathrm{~h}$ at $4{ }^{\circ} \mathrm{C}$. Primary antibodies were diluted in blocking buffer at these dilutions: von Willebrand Factor (vwf; 1:400), GATA4 (1:100), cardiac troponin I (1:400), CD163 (1:400), and CD68 (1:100). After thorough washing of tissue sections with $1 \times$ PBS, they were incubated with secondary antibody (Alexa Fluor 488 or $647-1: 200$ ) for $2 \mathrm{~h}$ and washed again. The nuclei staining of tissue sections were carried out using DAPI mounting medium, and fluorescence images were captured. Negative controls were made following the same protocol without the primary antibodies. To characterize the vessels, sections were 
stained with rabbit anti- $a$-SMA $(1: 100)$ and mouse anti-CD31 $(1: 100)$ at $4{ }^{\circ} \mathrm{C}$ overnight, followed by secondary anti-rabbit Alexa Fluor 647 and anti-mouse Alexa Fluor 488 to detect both the staining.

\subsection{Image Analysis and Quantification.}

The quantification of vessels was carried out in the MI $(n=6), 300 \mu \mathrm{m}$ dPMS on MI ( $n=6)$, and $600 \mu \mathrm{m}$ dPMS on MI $(n=6)$ groups at week 1, week 2, and week 4. For each rat heart, six regions (four images in the infarcted area and two images in the border region/suture area) were captured at $20 \times$ and measured using the imagescope software (Leica). Tubular structures positive for $a \mathrm{SMA} / \mathrm{CD} 31$ staining were identified as vessels and further classified into (a) arterioles and post capillary veins, (b) small artery and venule, (c) small veins, (d) artery, and (e) vein based on their size and shape. Average vessels were counted for each region and represented in per $\mathrm{mm}^{2}$ area.

Macrophages were detected by CD68 (total macrophage) and CD163 (M2 macrophage) staining in the MI $(n=6), 300 \mu \mathrm{m}$ dPMS on MI $(n=6)$, and $600 \mu \mathrm{m}$ dPMS on MI $(n=6)$ groups at week 1 , week 2 , and week 4 . For each slide, six regions (four images in the infarcted area and two images in the border zone) were captured at 20× magnification. Cells showing positive staining with DAPI nuclei were counted as one cell, and all of the images were counted in double-blinded fashion using the ImageJ. To assess the M2 macrophage population among total macrophages, the ratio of $\mathrm{CD} 163^{+} / \mathrm{CD} 68^{+}$cells was calculated. Macrophages were represented in per $\mathrm{mm}^{2}$ area.

\subsection{Statistics.}

Results are expressed as the mean \pm standard deviation for each group. Statistical software IBM SPSS 24 was used for the analysis of this study. Sample size calculation using post hoc power analysis revealed that the minimal sample size of five per group is required to achieve the power of $>80 \%$ with $a$ of 0.05 , while comparing three groups at three different time points. To compare multiple groups, analysis of variance for the parametric distribution and Kruskal-Wallis for nonparametric distribution were used along with Dunnett's test for a significance value. Comparison between two groups were conducted using the $t$-test or Mann-Whitney test depending on the data distribution. $p<0.05$ was considered as statistically significant.

\section{RESULTS}

\subsection{Implantation of dPMS in a Rat Model of Acute MI.}

Animal studies were performed following our experimental plan (Figure 1A). Decellularized porcine myocardium ( 1 $\mathrm{cm} \times 1 \mathrm{~cm}$; length $\mathrm{x}$ width) was characterized after decellularization process (Supporting Information, Figure S1) to ensure the sufficient removal of cells and then used to obtain dPMSs with the desired thicknesses (300 and 600 $\mu \mathrm{m})$. The obtained dPMSs maintained their physical integrity and ECM fiber orientation (Figure 1B,C). Immediately after LAD ligation, dPMS was placed onto the MI region and stabilized by suturing the edges (Figure 1D,E). After 7 days of implantation, when the heart was harvested for further examination, a clear volume increase of the implanted dPMS was 
observed (Figure 1F,I). Swelling of dPMS gradually decreased from week 1 to week 2 (Figure 1G,J). Significant dPMS degradation was found 4 weeks after implantation (Figure $1 \mathrm{H}, \mathrm{K})$. It was noticed that the implanted dPMS could firmly attach to host myocardium from week 1 to week 4 and physically integrated with the host tissue (Figure 2B,C). The patched dPMS prevented the progress of LV wall thinning caused by the MI (Figure 2A,F). The LV wall thickness of myocardium that received dPMS (600 $\mu$ m thickness) was significantly higher than the control group 4 weeks after treatment (Figure 2F).

\subsection{Infiltration of Host Cells into dPMS.}

Masson's trichrome staining reveals the morphology of rat myocardium after MI and dPMS treatments (Figure 2A-C). A large number of host cells was found to infiltrate into the dPMS (both 300 and $600 \mu \mathrm{m}$ thickness groups) after implantation, and a significant number of vessel structures was identified in the dPMS and infarcted area (Figure 2D,E). The formation of new vessels was further confirmed by immunostaining using aSMA, CD31, and vwf (Figure 3A-F). The quantification of vessels showed a significantly higher number of vessels present in the groups implanted with dPMS (both 300 and $600 \mu \mathrm{m}$ thickness) than in the MI group at week 1,2, and 4. (Figure 4A). We did not observe any significant alteration in the composition of different vessel types between the MI and MI with dPMS groups at any tested time point (Figure 4B). No positive staining of cardiac markers (GATA4 and cT1) was observed in the treatment groups (Figure 3G-L). The presence of macrophage in sham and MI area was checked using macrophage markers CD68 (total macrophage) and CD163 (M2 macrophage) (Figure 5A-H). We found a significantly higher density of M2 macrophages in the groups treated with dPMSs as compared to the control groups (sham and MI without treatment) from week 1 to week 4 (Figure 5I). The number of CD163 ${ }^{+} \mathrm{M} 2$ cells in the treatment groups (MI treated with $300 \mu \mathrm{m}$ dPMS, MI treated with $600 \mu \mathrm{m}$ dPMS) and control group (MI only) were $390 \pm 121.9,353.3 \pm 78.5$, and $92.2 \pm 67.2$ at week 1, 334.3 $\pm 91.6,341 \pm 111.2$, and $87 \pm 40.6$ at week 2 , and $336.3 \pm 118.7,356.3 \pm 111.2$, and 66.5 \pm 36.4 at week 4 , respectively. The ratio of $\mathrm{CD} 163^{+} / \mathrm{CD} 68^{+}$macrophages showed that an MI treated with 300 and $600 \mu \mathrm{m}$ dPMSs had a significantly higher ratio of M2 macrophages $(0.83 \pm 0.002$ and $0.98 \pm 0.07$, respectively) as compared to the MI group $(0.33 \pm 0.02)$ at week 4 (Figure 5J). As compared to the MI and MI treated with dPMS groups, the sham group demonstrated significantly fewer M2 macrophages and total macrophages present from week 1 to week 4 . However, we found that the ratio of M2 macrophages to total macrophages in dPMS-treated groups was similar to the sham groups from week 1 to week 4.

\subsection{Improvement of Cardiac Function by dPMS.}

Cardiac function was assessed using echocardiography preoperatively (baseline), 2 weeks and 4 weeks after surgery. As cardiac function indicators, LV ejection fraction (LVEF) and fractional shortening (FS) were determined by echocardiographic images and compared between groups. The baseline LVEF and FS were $71.9 \pm 1$ and $43.8 \pm 2.3 \%$, respectively. After the MI, the cardiac function of all rats was compromised. For the control group (MI without treatment), a decline in the cardiac function continued over the 4 weeks. After 4 weeks, LVEF and FS of the infarcted hearts were significantly reduced to $42 \pm 4.3$ and 19.4 $\pm 6.7 \%$, respectively. M-mode echocardiographic images showed the LV wall motion after 
treatment (Figure 6A). Contraction of the LV wall was observed to improve in the treatment group (300 and $600 \mu \mathrm{m}$ dPMS) at week 4 as compared to the MI group (Figure 6A). Left ventricle ejection fractions in MI $+300 \mu \mathrm{m}$ dPMS and MI $+600 \mu \mathrm{m}$ dPMS groups were significantly improved to $62.8 \pm 9$ and $59.2 \pm 8 \%$, respectively, at week 4 compared to the MI group (Figure 6B). Fractional shortening in MI $+600 \mu \mathrm{m}$ dPMS group was significantly improved to $33.2 \pm 5.7 \%$ compared to the MI group after 4 weeks of transplantation (Figure $6 \mathrm{C})$.

\section{DISCUSSION}

The feasibility of using a cardiac patch to treat an MI has been proven by many research groups. ${ }^{17-19}$ Both cell-containing cardiac patches and acellular patches have been explored and demonstrated contributions to cardiac repair. ${ }^{13,20}$ For cell-containing patches, the ultimate goal is to replace the dead cardiomyocytes, lost during an MI, with the new functional cardiomyocytes coming or deriving from patch-derived cells. However, the extremely poor survival and low engraftment rate of the delivered cells remain the major hurdles in implementing this strategy. ${ }^{6}$ For acellular patches, it has been shown that the main mechanisms behind their therapeutic benefits in cardiac repair are related to either the biomolecules (e.g., therapeutic miRNA, cytokines, growth factors) delivered by the acellular patch or the mechanical support provided by the patch to help enhance LV contractility. ${ }^{21,22}$ Many types of synthetic materials including poly(lactic-co-glycolic acid), poly(ethylene glycol), polycaprolactone have been explored to treat MI and demonstrated unique advantages such as their off-the-shelf availability, broad tunability in mechanical properties and degradation rate, and great scale-up capability. ${ }^{23,24}$ However, compared with natural biomaterials (e.g., dPMS), synthetic materials generally lack cell interactions, often elicit elevated host inflammatory response, and suffer from problems, such as host compliance and integration. $^{25,26}$

In this study, we examined the in vivo effects of using a decellularized porcine myocardium slice (dPMS) as an acelluar cardiac patch to treat MI. Through our previous in vitro studies, we have shown that the thickness affects the material properties of dPMS and their interactions with the seeded cells. ${ }^{16,27}$ Among all of the thicknesses, we explored ranging from 300 to $900 \mu \mathrm{m}$, we identified 300 and $600 \mu \mathrm{m}$ as our targeted thicknesses to fabricate the acellular patch for the following reasons: (1) both 300- and 600- $\mu \mathrm{m}$-thick dPMSs maintain great physical integrity and show acceptable in vitro degradation rate after seeding with cells; (2) 300- $\mu \mathrm{m}$-thick dPMS allows direct diffusion of oxygen and nutrients through the scaffold, which enables the high survival rate of seeded cells; and (3) $600-\mu \mathrm{m}$-thick dPMS preserves the mechanical properties of native cardiac tissue, which could aid the deteriorating LV function after an MI. Therefore, in the present study, dPMSs with two thicknesses ( 300 and $600 \mu \mathrm{m}$ ) have been selected as the treatment groups to compare their therapeutic outcomes.

Our results demonstrate the unique advantages of dPMS serving as an acellular cardiac patch. First, dPMS (both 300 and $600 \mu \mathrm{m}$ thicknesses) firmly attached to the host tissue after implantation. Unlike many other cardiac patches that need to add extra adhesives such as fibrin glue between the patch and the host tissue to help bind the patch onto myocardium, ${ }^{28}$ 
dPMS adhered by itself to the transplantation site during implantation and maintained physical integration with the host after implantation. For all of the rats that received dPMS treatment, when we harvested the heart tissue at planned time points $(1,2$, and 4 weeks after treatment), we could clearly see that the dPMS remained as a whole piece integrated with the host myocardium. Histological analysis also confirmed that there was no gap between the host myocardium and the engrafted patch. Second, dPMS implantation improved the cardiac function. Both 300- and 600- $\mu \mathrm{m}$-thick dPMSs increased LV contractility after treatment. However, as compared with 300- $\mu$ m-thick dPMS, the 600- $\mu \mathrm{m}$-thick dPMS better prevented LV wall thinning and improved FS when assessed at 4 weeks after implantation. Finally, dPMS promoted in vivo vascularization from infiltrated host cells. Our dPMS was derived from decellularized porcine cardiac tissue and contained no detectable cells when implanted as an acellular patch. Our results revealed that a large number of host cells infiltrated into dPMS and formed different types of vessels 7 days after implantation. A similar phenomenon has also been reported by other groups using ECM scaffold for the cardiac repair. ${ }^{29}$ The retention of various growth factors in the decellularizd tissue has been reported by many researchers, which contributes to the bioactivity of acellular ECM as a biological scaffold. ${ }^{9,30}$ However, compared with native tissue, the amount of retained growth factors in decellularized tissue is significantly reduced. For example, the amount of angiogenic factor VEGF in porcine small intestine submucosa (SIS) ECM has been reduced to $0.77 \mathrm{ng} / \mathrm{g}$ after decellularization using urea/heparin solution for $24 \mathrm{~h} .{ }^{31}$

Future studies will help to further improve the therapeutic outcomes of dPMS as a cardiac patch. After 4 weeks of implantation, a significant degradation of dPMS was observed especially for the 300- $\mu \mathrm{m}$-thick dPMS treatment group, which may compromise the longterm benefits of dPMS. We plan to slow the in vivo degradation of dPMS using a natural crosslinker in future studies and test the effects of tuning degradation rate on cardiac function improvement. ${ }^{32}$ It needs to be noted that compared with the mechanical property of native rat myocardium, the dPMSs preserve the anisotropic nonlinear behavior of cardiac tissue and have significantly increased stiffness and tensile modulus in both longitudinal and circumferential directions. ${ }^{16,33}$ Future tuning of the dPMS mechanical properties to better mimic the native rat myocardium may further improve the therapeutic outcomes in rat MI model. The critical roles of macrophages in cardiac repair and regeneration have been gradually recognized in recent years. ${ }^{34}$ In this study, we found that the majority of infiltrated host cells expressed macrophages markers (CD163, CD68), and the presence of M2 type $\left(\mathrm{CD} 163^{+}\right)$macrophages significantly increased in treatment groups 4 weeks after the implantation. This significant number of infiltrated inflammatory cells after dPMS treatment suggest an unsuppressed post-MI inflammation. It has been reported that inflammatory responses after MI contribute to cardiomyocytes death, cardiac fibrosis, and pathological ventricular remodeling. ${ }^{35,36} \mathrm{We}$ anticipate that in future, the inclusion of anti-inflammation strategies in dPMS treatment will further improve the therapeutic outcomes. Also, our future studies will closely examine the interactions between the implanted dPMS and the various types of infiltrated host macrophages, which will help elucidate the full picture of the immune response after dPMS implantation. In addition, for all of the tissues we harvested and carefully analyzed, we did not find any cells in the implanted dPMS that expressed cardiac markers (GATA4, cT1). Adding viable functional cardiomyocytes to the injured 
myocardium is the eventual cure for MI. In future studies, we will use our dPMS to exploit tissue engineering strategies such as recruiting endogenous cardiac cells and combining with cell delivery to achieve this ultimate goal.

\section{CONCLUSIONS}

We tested using decellularized porcine myocardial slice (dPMS) as an acellular cardiac patch to treat the acutely infarcted heart in a rat MI model. We found that dPMS attached firmly to the infarcted region and integrated with the host myocardium. A high number of host cells was found to infiltrate into dPMS, and a high number of M2 (anti-inflammatory) macrophages was present. dPMS promoted neo-vascularization within dPMS and infarcted areas after 1 week of transplantation. dPMS also improved ejection fraction and fractional shortening of the infarcted heart 4 weeks after implantation. These results demonstrate the benefits of using dPMS as an acellular cardiac patch to treat MI.

\section{Supplementary Material}

Refer to Web version on PubMed Central for supplementary material.

\section{ACKNOWLEDGMENTS}

We are thankful for the financial support from the National Institute of Health (1R15HL22929) and the American Heart Association (19AIREA34400087).

\section{REFERENCES}

(1). Sutton MG; Sharpe N Left Ventricular Remodeling after Myocardial Infarction: Pathophysiology and Therapy. Circulation 2000, 101, 2981-2988. [PubMed: 10869273]

(2). Visser CA Left Ventricular Remodelling after Myocardial Infarction: Importance of Residual Myocardial Viability and Ischaemia. Heart 2003, 89, 1121-1122. [PubMed: 12975390]

(3). Ibàñez B; Heusch G; Ovize M; Van de Werf F Evolving Therapies for Myocardial Ischemia/ reperfusion Injury. J. Am. Coll. Cardiol 2015, 65, 1454-1471. [PubMed: 25857912]

(4). Godier-Furnémont AFG; Martens TP; Koeckert MS; Wan L; Parks J; Arai K; Zhang G; Hudson B; Homma S; Vunjak-Novakovic G Composite Scaffold Provides a Cell Delivery Platform for Cardiovascular Repair. Proc. Natl. Acad. Sci. U.S.A 2011, 108, 7974-7979. [PubMed: 21508321]

(5). Zhang J Engineered Tissue Patch for Cardiac Cell Therapy. Curr. Treat. Options Cardiovasc. Med 2015, 17, No. 399.

(6). Hoover-Plow J; Gong Y Challenges for Heart Disease Stem Cell Therapy. Vasc. Health Risk Manage 2012, 8, 99-113.

(7). Raval AN; Kamp TJ; Hogle LF Cellular Therapies for Heart Disease: Unveiling the Ethical and Public Policy Challenges. J. Mol. Cell. Cardiol 2008, 45, 593-601. [PubMed: 18155721]

(8). Penicka M; Widimsky P; Kobylka P; Kozak T; Lang O Images in Cardiovascular Medicine. Early Tissue Distribution of Bone Marrow Mononuclear Cells after Transcoronary Transplantation in a Patient with Acute Myocardial Infarction. Circulation 2005, 112, e63-e65. [PubMed: 16043651]

(9). Crapo PM; Gilbert TW; Badylak SF An Overview of Tissue and Whole Organ Decellularization Processes. Biomaterials 2011, 32, 3233-3243. [PubMed: 21296410]

(10). Petersen TH; Calle EA; Colehour MB; Niklason LE Matrix Composition and Mechanics of Decellularized Lung Scaffolds. Cells Tissues Organs 2012, 195, 222-231. [PubMed: 21502745]

(11). Moroni F; Mirabella T Decellularized Matrices for Cardiovascular Tissue Engineering. Am. J. Stem Cells 2014, 3, 1-20. [PubMed: 24660110] 
(12). Shahabipour F; Banach M; Johnston TP; Pirro M; Sahebkar A Novel Approaches toward the Generation of Bioscaffolds as a Potential Therapy in Cardiovascular Tissue Engineering. Int. J. Cardiol 2017, 228, 319-326. [PubMed: 27866022]

(13). Robinson KA; Li J; Mathison M; Redkar A; Cui J; Chronos NAF; Matheny RG; Badylak SF Extracellular Matrix Scaffold for Cardiac Repair. Circulation 2005, 112, I135-I143. [PubMed: 16159805]

(14). Ramos CMG; Francisco JC; Olandoski M; de Carvalho KAT; Cunha R; Erbano BO; Jorge LF; Baena CP; do Amaral VF; Noronha L; Macedo RM; Faria-Neto JR; Guarita-Souza LC Myocardial Regeneration after Implantation of Porcine Small Intestinal Submucosa in the Left Ventricle. Braz. J. Cardiovasc. Surg 2014, 29, 202-213.

(15). Mewhort HEM; Svystonyuk DA; Turnbull JD; Teng G; Belke DD; Guzzardi DG; Park DS; Kang S; Hollenberg MD; Fedak PWM Bioactive Extracellular Matrix Scaffold Promotes Adaptive Cardiac Remodeling and Repair. JACC Basic Transl. Sci 2017, 2, 450-464. [PubMed: 30062163]

(16). Shah M; Kc P; Copeland KM; Liao J; Zhang G A Thin Layer of Decellularized Porcine Myocardium for Cell Delivery. Sci. Rep 2018, 8, No. 16206.

(17). Wang Q; Yang H; Bai A; Jiang W; Li X; Wang X; Mao Y; Lu C; Qian R; Guo F; Ding T; Chen $\mathrm{H}$; Chen S; Zhang J; Liu C; Sun N Functional Engineered Human Cardiac Patches Prepared from Nature's Platform Improve Heart Function after Acute Myocardial Infarction. Biomaterials 2016, 105, 52-65. [PubMed: 27509303]

(18). Ye L; Zimmermann W-H; Garry DJ; Zhang J Patching the Heart: Cardiac Repair from within and Outside. Circ. Res 2013, 113, 922-932. [PubMed: 24030022]

(19). Zhang J; Zhu W; Radisic M; Vunjak-Novakovic G Can We Engineer a Human Cardiac Patch for Therapy? Circ. Res 2018, 123, 244-265. [PubMed: 29976691]

(20). Gao L; Gregorich ZR; Zhu W; Mattapally S; Oduk Y; Lou X; Kannappan R; Borovjagin AV; Walcott GP; Pollard AE; Fast VG; Hu X; Lloyd SG; Ge Y; Zhang J Large Cardiac Muscle Patches Engineered From Human Induced-Pluripotent Stem Cell-Derived Cardiac Cells Improve Recovery From Myocardial Infarction in Swine. Circulation 2018, 137, 1712-1730. [PubMed: 29233823]

(21). Svystonyuk DA; Mewhort HEM; Fedak PWM Using Acellular Bioactive Extracellular Matrix Scaffolds to Enhance Endogenous Cardiac Repair. Front. Cardiovasc. Med 2018, 5, No. 35.

(22). Serpooshan V; Zhao M; Metzler SA; Wei K; Shah PB; Wang A; Mahmoudi M; Malkovskiy AV; Rajadas J; Butte MJ; Bernstein D; Ruiz-Lozano P The Effect of Bioengineered Acellular Collagen Patch on Cardiac Remodeling and Ventricular Function Post Myocardial Infarction. Biomaterials 2013, 34, 9048-9055. [PubMed: 23992980]

(23). Lam MT; Wu JC Biomaterial Applications in Cardiovascular Tissue Repair and Regeneration. Expert Rev. Cardiovasc. Ther 2012, 10, 1039-1049. [PubMed: 23030293]

(24). Kai D; Wang Q-L; Wang H-J; Prabhakaran MP; Zhang Y; Tan Y-Z; Ramakrishna S Stem CellLoaded Nanofibrous Patch Promotes the Regeneration of Infarcted Myocardium with Functional Improvement in Rat Model. Acta Biomater. 2014, 10, 2727-2738. [PubMed: 24576580]

(25). Pascual-Gil S; Garbayo E; Díaz-Herráez P; Prosper F; Blanco-Prieto MJ Heart Regeneration after Myocardial Infarction Using Synthetic Biomaterials. J. Controlled Release 2015, 203, 23-38.

(26). Singelyn JM; Christman KL Injectable Materials for the Treatment of Myocardial Infarction and Heart Failure: The Promise of Decellularized Matrices. J. Cardiovasc. Transl. Res 2010, 3, 478486. [PubMed: 20632221]

(27). Kc P; Shah M; Liao J; Zhang G Prevascularization of Decellularized Porcine Myocardial Slice for Cardiac Tissue Engineering. ACS Appl Mater. Interfaces 2017, 9, 2196-2204. [PubMed: 28029762]

(28). Melhem M; Jensen T; Reinkensmeyer L; Knapp L; Flewellyn J; Schook L A Hydrogel Construct and Fibrin-Based Glue Approach to Deliver Therapeutics in a Murine Myocardial Infarction Model. J. Vis. Exp 2015, No. 100, No. e52562.

(29). Sarig U; Sarig H; de-Berardinis E; Chaw S-Y; Nguyen EBV; Ramanujam VS; Thang VD; AlHaddawi M; Liao S; Seliktar D; Kofidis T; Boey FY; Venkatraman SS; Machluf M Natural Myocardial ECM Patch Drives Cardiac Progenitor Based Restoration Even after Scarring. Acta Biomater. 2016, 44, 209-220. [PubMed: 27545814] 
(30). Taipale J; Keski-Oja J Growth Factors in the Extracellular Matrix. FASEB J. 1997, 11, 51-59. [PubMed: 9034166]

(31). Hodde JP; Record RD; Liang HA; Badylak SF Vascular Endothelial Growth Factor in PorcineDerived Extracellular Matrix. J. Endothelial Cell Res 2001, 8, 11-24.

(32). Jeffords ME; Wu J; Shah M; Hong Y; Zhang G Tailoring Material Properties of Cardiac Matrix Hydrogels to Induce Endothelial Differentiation of Human Mesenchymal Stem Cells. ACS Appl. Mater. Interfaces 2015, 7, 11053-11061. [PubMed: 25946697]

(33). Sirry MS; Butler JR; Patnaik SS; Brazile B; Bertucci R; Claude A; McLaughlin R; Davies NH; Liao J; Franz T Characterisation of the Mechanical Properties of Infarcted Myocardium in the Rat under Biaxial Tension and Uniaxial Compression. J. Mech. Behav. Biomed. Mater 2016, 63, 252-264. [PubMed: 27434651]

(34). Zlatanova I; Pinto C; Silvestre J-S Immune Modulation of Cardiac Repair and Regeneration: The Art of Mending Broken Hearts. Front. Cardiovasc. Med 2016, 3, No. 40.

(35). Kim YS; Ahn Y Functional Relevance of Macrophage-Mediated Inflammation to Cardiac Regeneration. Chonnam Med. J 2018, 54, 10-16. [PubMed: 29399560]

(36). Ma Y; Mouton AJ; Lindsey ML Cardiac Macrophage Biology in the Steady-State Heart, the Aging Heart, and Following Myocardial Infarction. Transl. Res 2018, 191, 15-28. [PubMed: 29106912] 
A
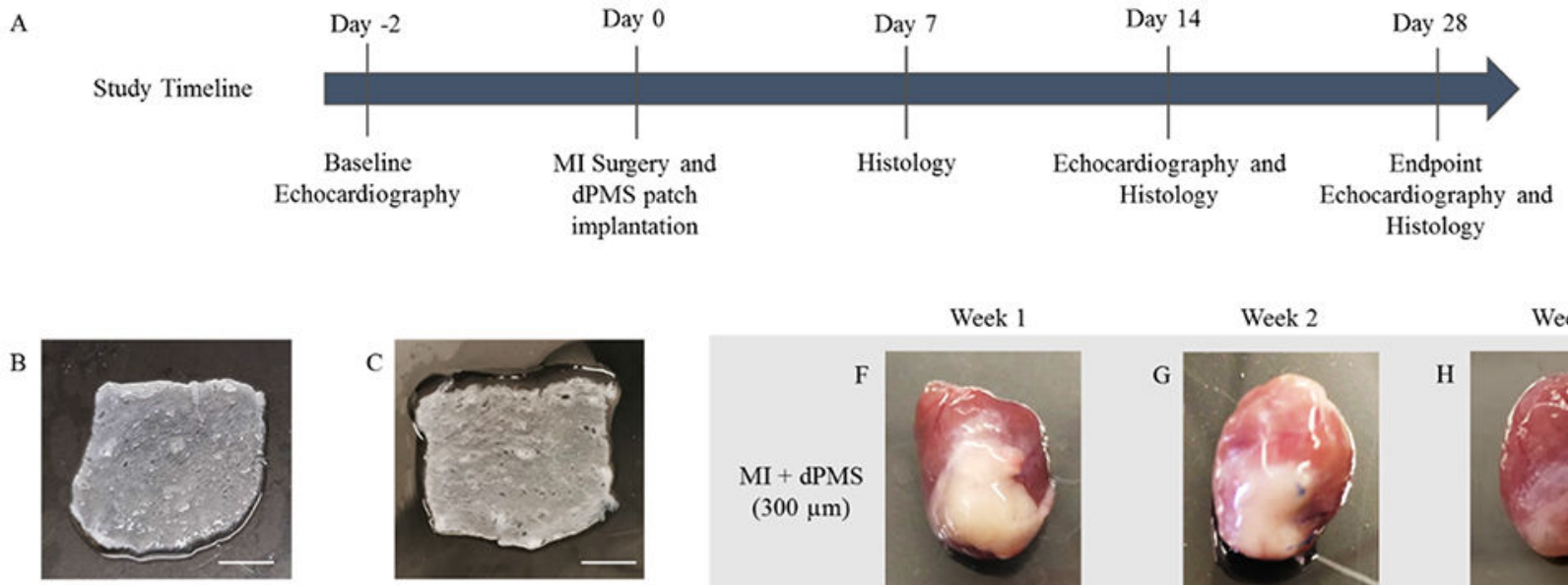

Week

Week 2

Week 4

D

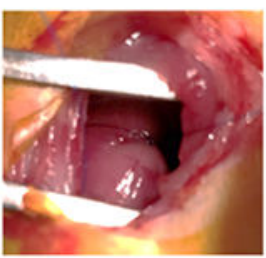

E

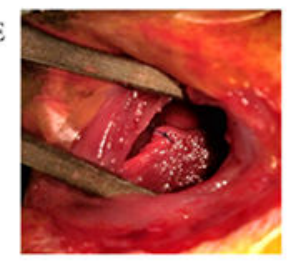

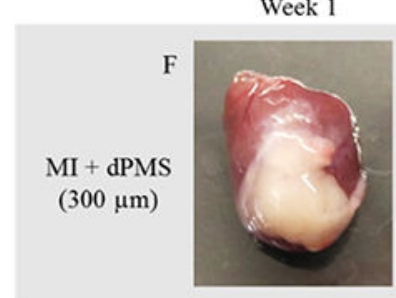

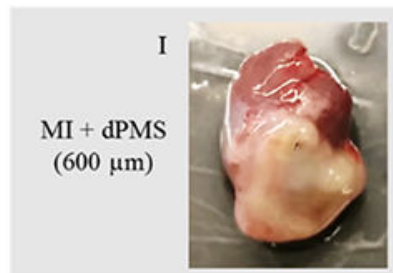

G

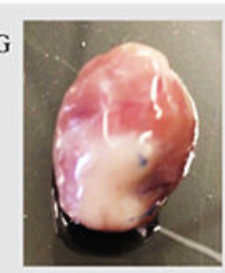

H

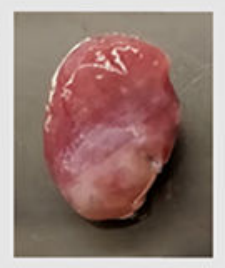

J

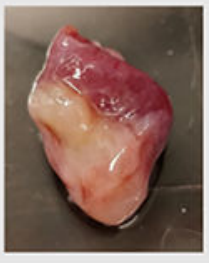

K

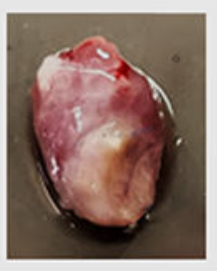

Figure 1.

Implantation of acellular dPMS: Schematic diagram of animal study timeline (A).

Representative images for $300 \mu \mathrm{m}$ (B) and $600 \mu \mathrm{m}$ (C) thick dPMSs. Infarct induced by LAD ligation (D) and implantation of the patch (E) shown during surgery. dPMSs integration (300 and $600 \mu \mathrm{m}$ ) to infarcted heart after week 1, week 2, and week 4 postsurgery $(\mathrm{F}-\mathrm{K})$. Scale bar: $3 \mathrm{~mm}$. 


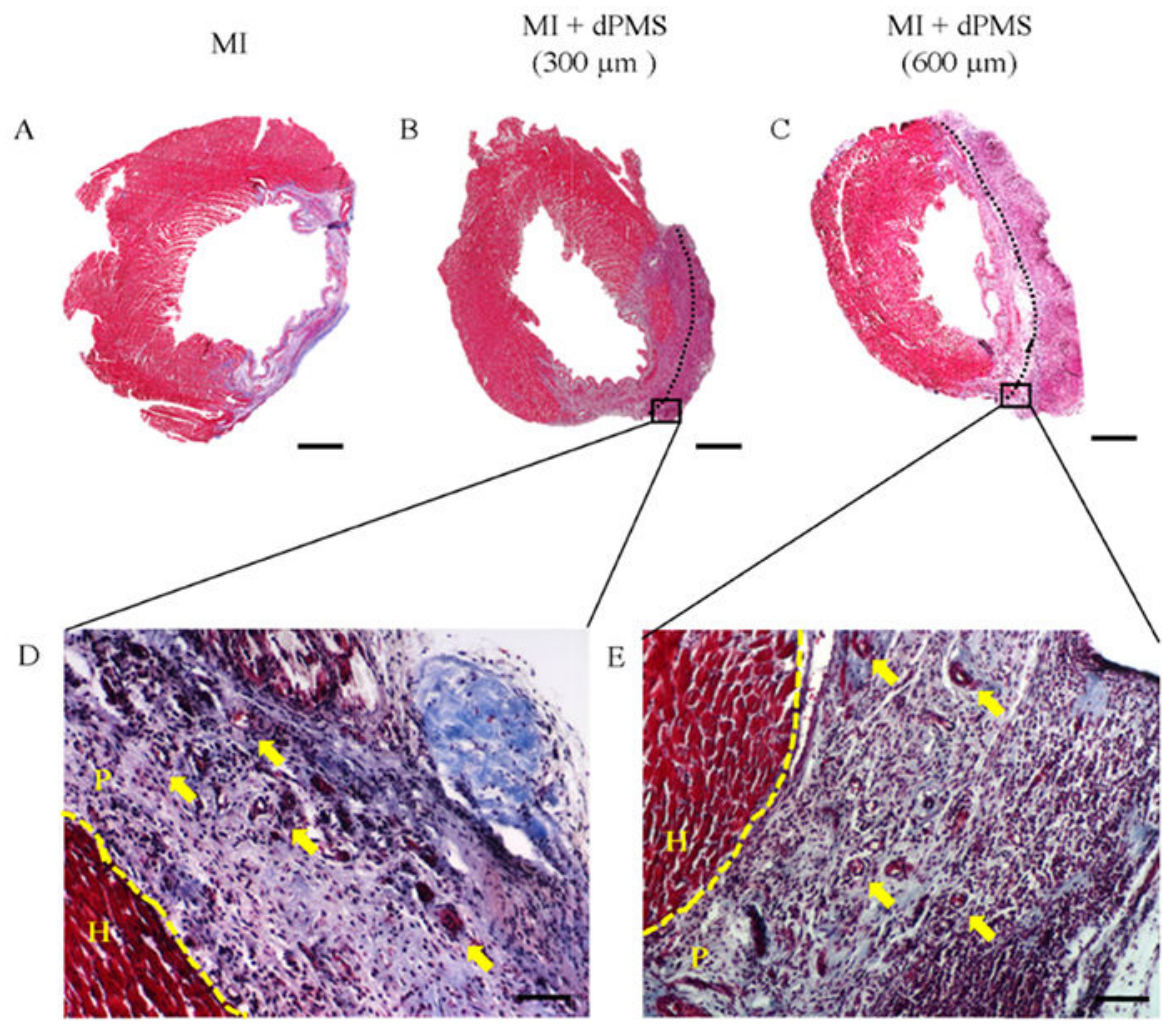

F

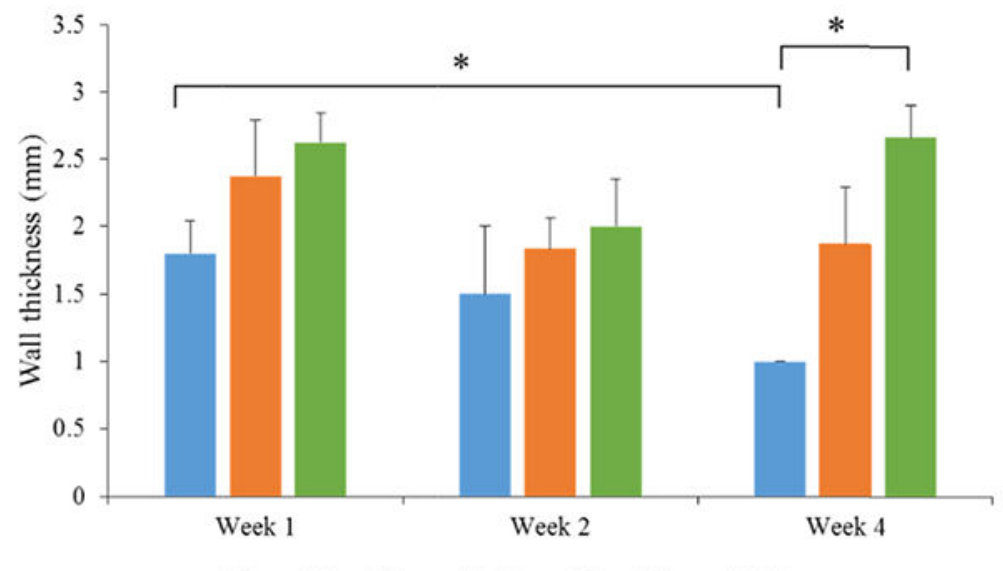

$=\mathrm{MI} \quad=\mathrm{MI}+300 \mu \mathrm{m} \mathrm{dPMS} \quad=\mathrm{MI}+600 \mu \mathrm{m} \mathrm{dPMS}$

Figure 2.

Histological evaluation. Masson's trichrome staining showing the entire transverse slice of infarct and dPMS implanted rat myocardium (A-C) and at higher magnification (D, E). The effect of dPMS implantation on LV wall thickness from week 1 to week 4 (F). Vessel-like structure in implanted dPMS shown by the arrows. Data were expressed as means $\pm \mathrm{SD}$ ( $n=$ 4 for each sample). *Represents the statistical significant difference with a $p<0.05$. Scale bar: A-C: $3 \mathrm{~mm}$. D, E: $100 \mu \mathrm{m}$. The dotted line is between heart $(\mathrm{H})$ and patch $(\mathrm{P})$. 
aSMA / CD31 / DAPI

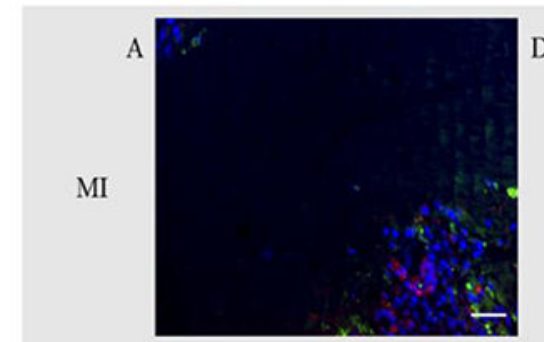

B
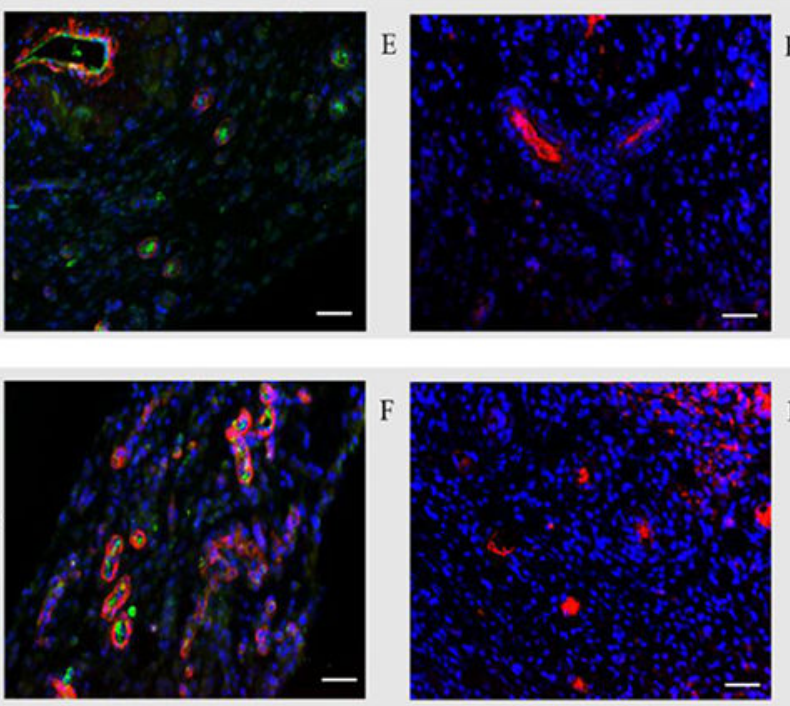
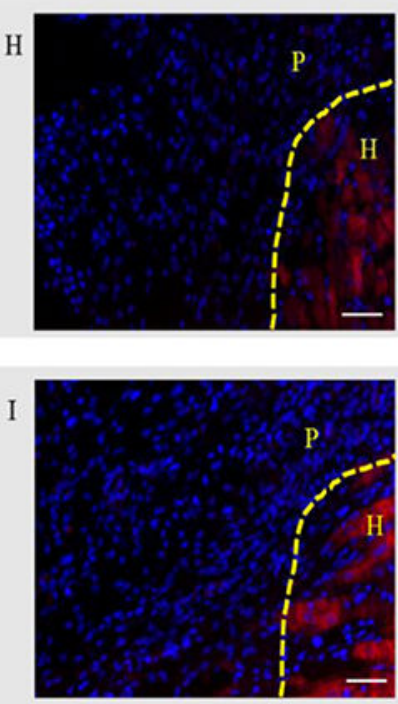

cTl / DAPI
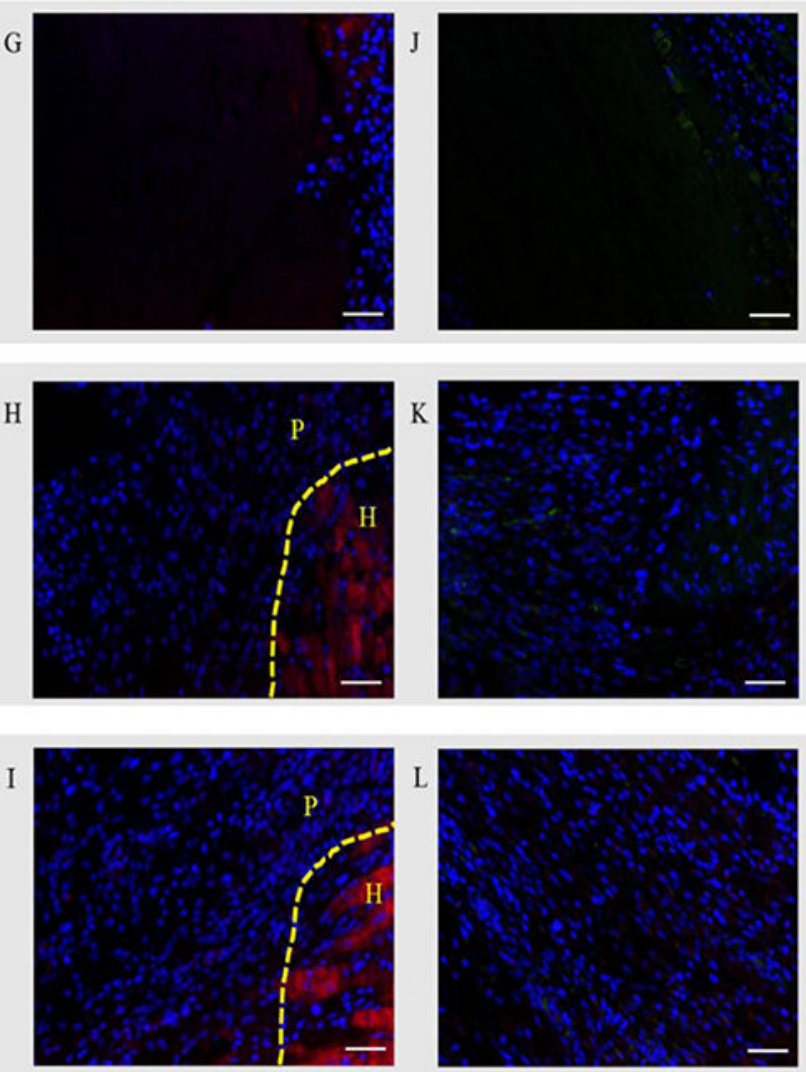

Figure 3.

Cardiovascular staining. MI and MI with dPMSs (300 and $600 \mu \mathrm{m})$ groups stained with vascular markers: $a \mathrm{SMA} / \mathrm{CD} 31(\mathrm{~A}-\mathrm{C})$ and vwf $(\mathrm{D}-\mathrm{F})$, cardiac markers: cardiac troponin I (cTI) (G-I) and GATA4 (J-L) post-surgery. Scale bar: $50 \mu \mathrm{m}$. The dotted line is between heart $(\mathrm{H})$ and patch $(\mathrm{P})$. 
A

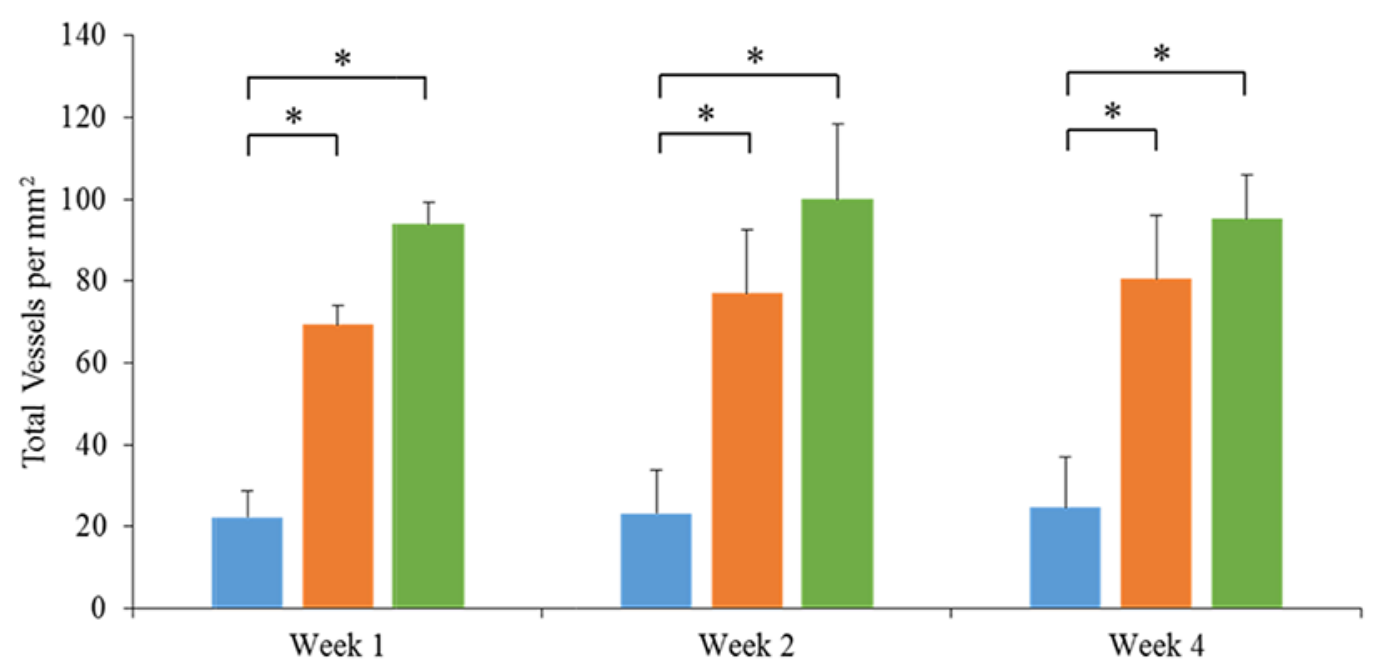

B

$\varpi \mathrm{MI} \quad \mathrm{MI}+\mathrm{dPMS}(300 \mu \mathrm{m}) \quad \square \mathrm{MI}+\mathrm{dPMS}(600 \mu \mathrm{m})$

MI

$\mathrm{MI}+300 \mu \mathrm{m} \mathrm{dPMS}$
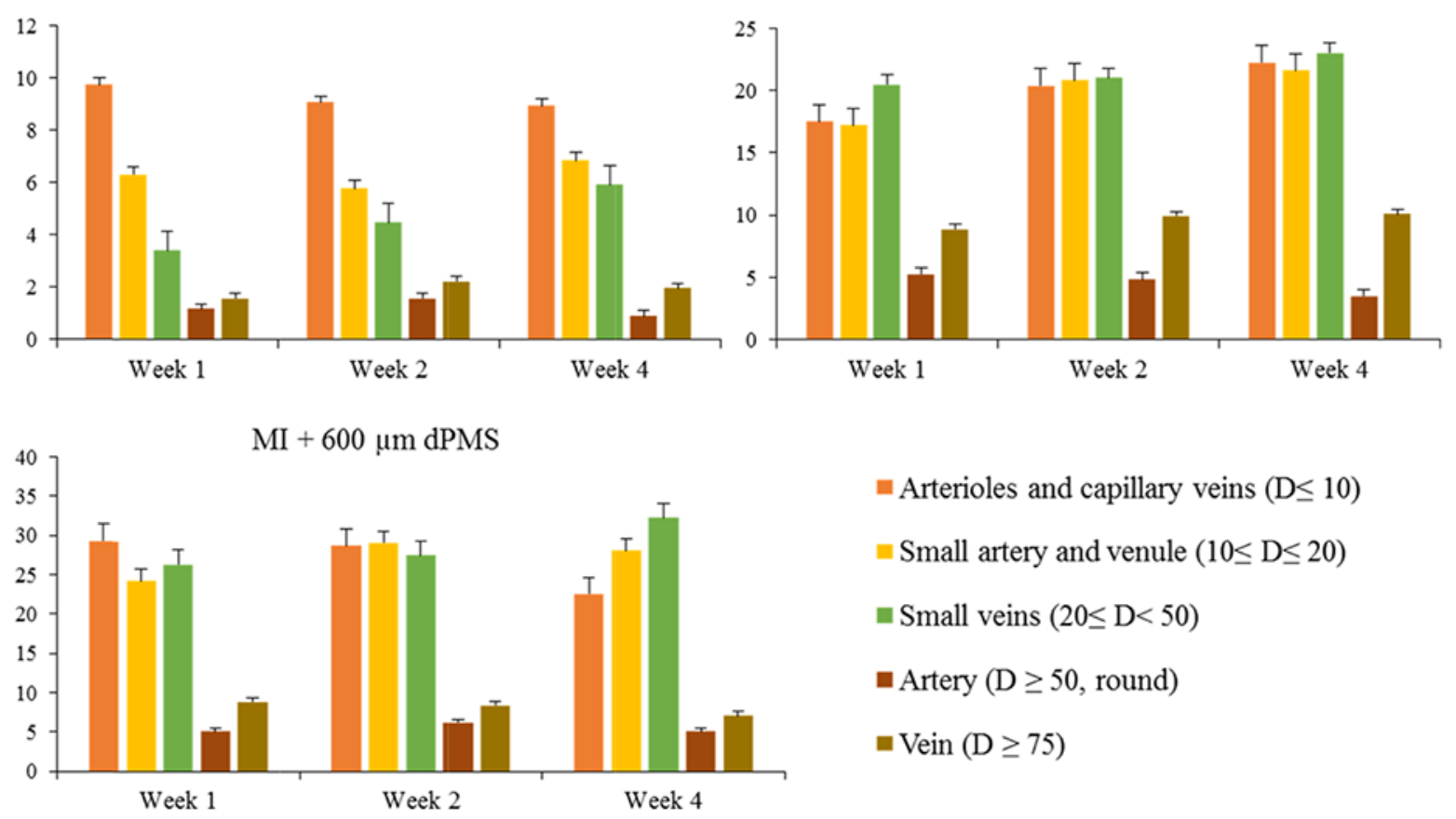

Figure 4.

MI region vessel density and composition analysis. The quantification of total vessels density in the MI region of the treatment and control groups at weeks 1, 2, and 4 postsurgery (A). The composition of different vessel types in the MI region from treatment and control groups is shown at week 1 , week 2 , and week 4 (B). Data were expressed as means \pm $\mathrm{SD}$ ( $n=6$ for each sample). * Represents the statistical significant difference with a $p<$ 0.05. D: diameter. 

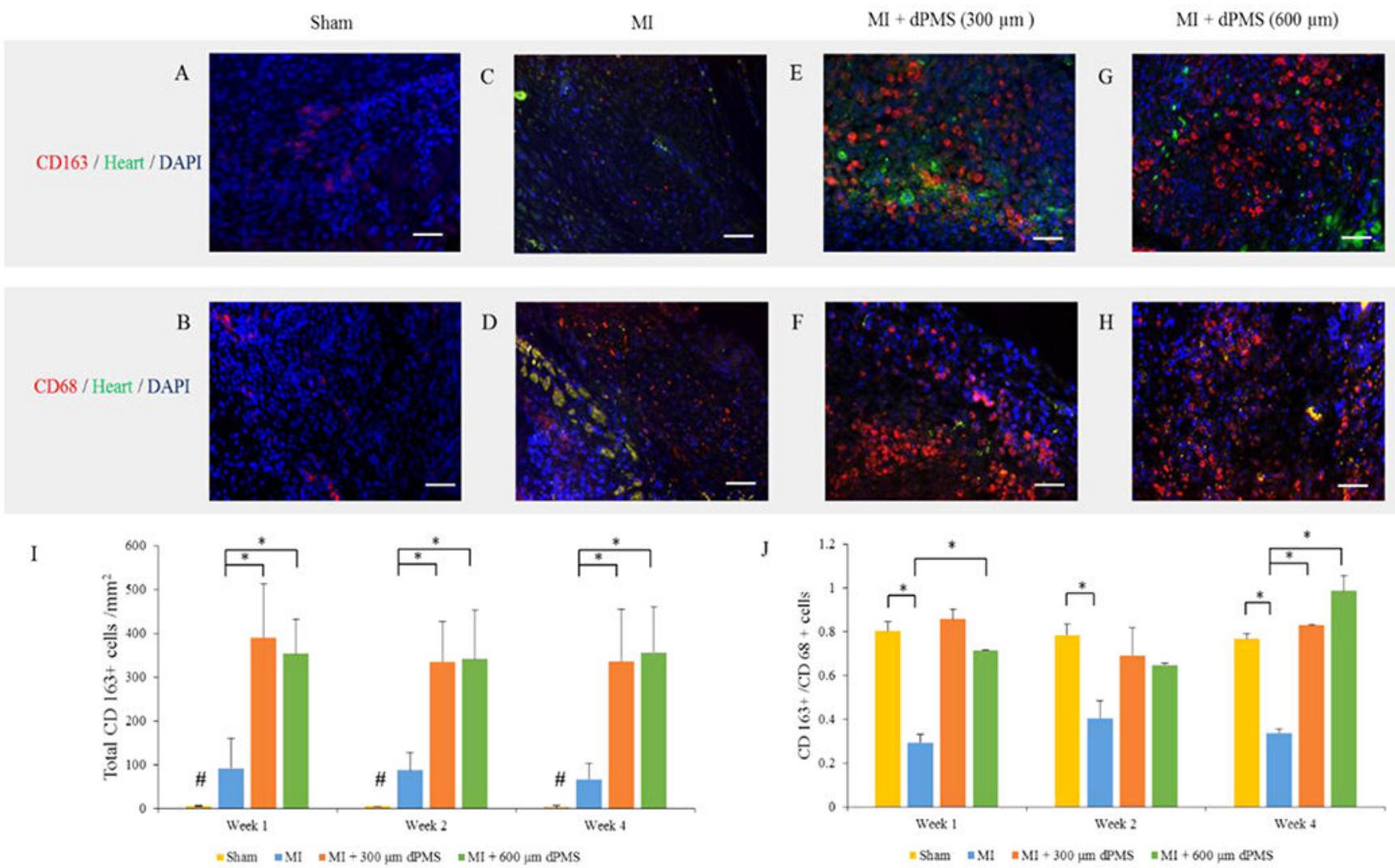

Figure 5.

Macrophage assessment. Immunofluorescence staining for total macrophages (CD68) and M2 macrophages (CD163) in sham, MI, and implanted dPMS groups (A-H). CD163 ${ }^{+}$cells quantified per $\mathrm{mm}^{2}$ area in sham, MI, and implanted dPMS groups after week 1, week 2, and week 4 (I). The ratio of $\mathrm{CD} 163^{+} / \mathrm{CD} 68^{+}$cells showing the distribution of M2 macrophages after week 1, week 2, and week 4 of implantation for each group (J). Data were expressed as means $\pm \mathrm{SD}$ ( $n=6$ for each sample). *Represents the statistical significant difference with a $p<0.05$. \#Represents the significant difference between study groups. Scale bar: $50 \mu \mathrm{m}$. 
A
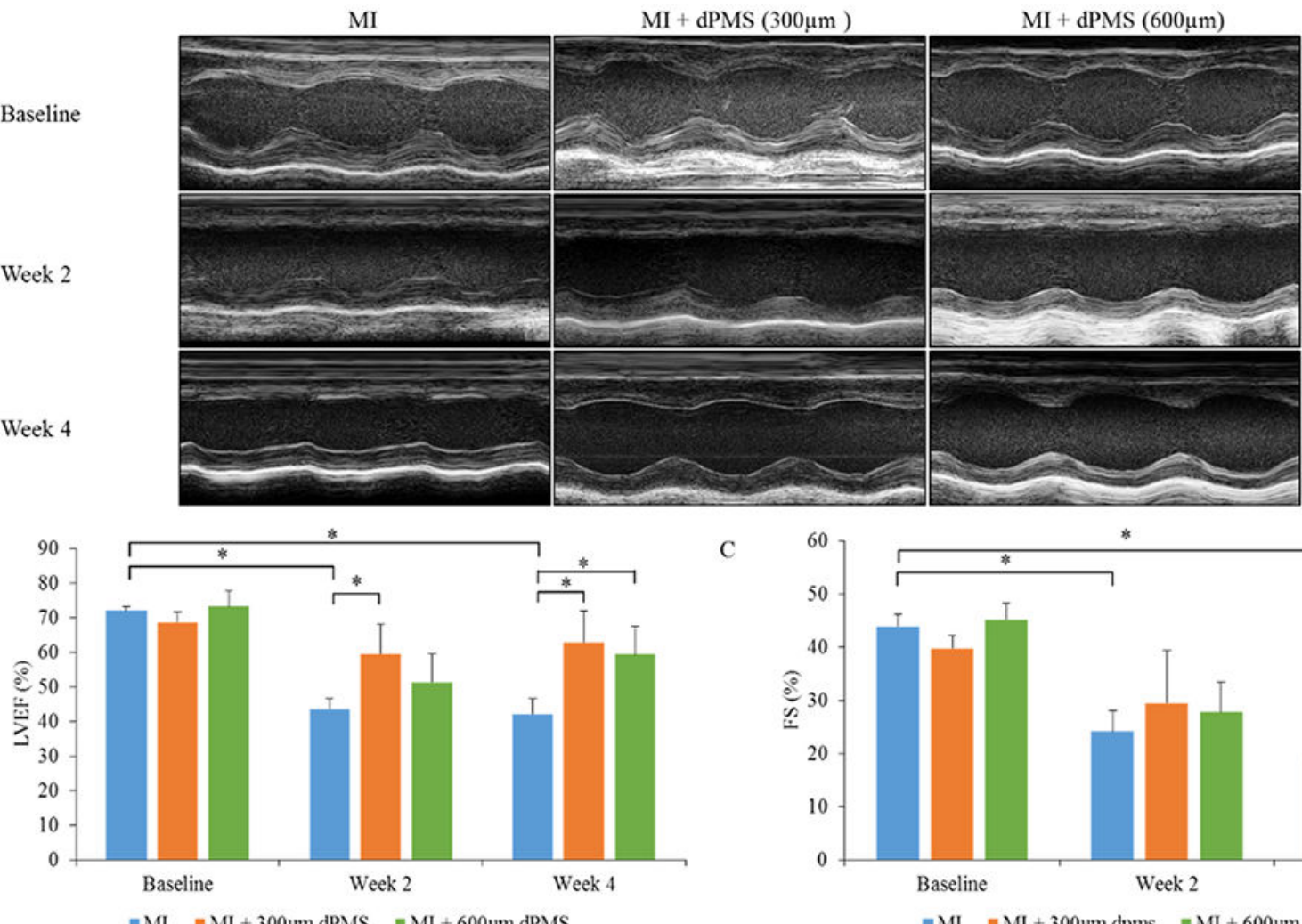

$\mathrm{C}$

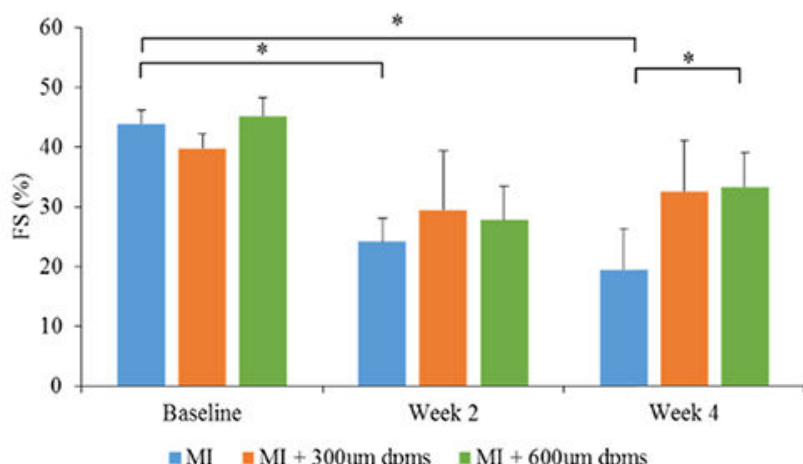

Figure 6.

Echocardiographic measurement. M-mode images showing the contractility of LV wall before surgery, 2 weeks post-op, and 4 weeks post-op for MI and MI with dPMSs (300 and $600 \mu \mathrm{m})$ groups (A). Percent left ventricle ejection fraction (LVEF) and fractional shortening (FS) in MI and MI with dPMSs (300 and $600 \mu \mathrm{m}$ ) groups before surgery, 2 weeks post-op, and 4 weeks post-op (B, C). Data were expressed as means $\pm \mathrm{SD}$ ( $n=5$ for each sample). *Represents the statistical significant difference with a $p<0.05$. 